\title{
Observable Windows for the QCD Axion Through the Number of Relativistic Species
}

\author{
Ricardo Z. Ferreira ${ }^{* *}$ and Alessio Notari ${ }^{\dagger}$ \\ Departament de Física Quàntica i Astrofisíca and Institut de Ciències del Cosmos (ICCUB), \\ Universitat de Barcelona, Martí i Franquès 1, 08028 Barcelona, Spain
}

(Received 18 January 2018; revised manuscript received 20 February 2018; published 11 May 2018)

\begin{abstract}
We show that when the QCD axion is directly coupled to quarks with $c_{i} / f \partial_{\mu} a \bar{q}_{i} \gamma^{\mu} \gamma^{5} q_{i}$, such as in Dine-Fischler-Srednicki-Zhitnitsky models, the dominant production mechanism in the early Universe at temperatures $1 \mathrm{GeV} \lesssim T \lesssim 100 \mathrm{GeV}$ is obtained via $q_{i} \bar{q}_{i} \leftrightarrow g a$ and $q_{i} g \leftrightarrow q_{i} a$, where $g$ are gluons. The production of axions through such processes is maximal around $T \approx m_{i}$, where $m_{i}$ are the different heavy quark masses. This leads to a relic axion background that decouples at such temperatures, leaving a contribution to the effective number of relativistic degrees of freedom, which can be larger than the case of decoupling happens the electroweak phase transition, $\Delta N_{\text {eff }} \lesssim 0.027$. Our prediction for the $t$ quark is $0.027 \leq \Delta N_{\text {eff }} \leq 0.036$ for $10^{6} \mathrm{GeV} \lesssim f / c_{t} \lesssim 4 \times 10^{8} \mathrm{GeV}$ and for the $b$ quark is $0.027 \leq \Delta N_{\text {eff }} \leq 0.047$ for $10^{7} \mathrm{GeV} \lesssim f / c_{b} \lesssim 3 \times 10^{8} \mathrm{GeV}$. For the $c$ quark the window can only be roughly estimated as $0.027<\Delta N_{\text {eff }} \lesssim \mathcal{O}(0.1)$, for $f / c_{c} \lesssim(2-3) \times 10^{8} \mathrm{GeV}$, since axions can still be partially produced in a regime of strong coupling, when $\alpha_{s} \gtrsim 1$. These contributions are comparable to the sensitivity of future CMB S4 experiments, thus opening an alternative window to detect the axion and to test the early Universe at such temperatures.
\end{abstract}

DOI: 10.1103/PhysRevLett.120.191301

Introduction.-The axion field, postulated for theoretical reasons [1,2], possesses a very rich phenomenology [3]. It has a coupling to gluons of the form $\alpha_{s} /(8 \pi f) a G \tilde{G}$, that can provide a compelling solution to the strong $C P$ problem [4], i.e., the fact that the total coefficient of the $C P$ violating operator $\theta /(8 \pi) G \tilde{G}$ has a coefficient consistent with zero in a vacuum with high precision, $|\theta| \lesssim$ $10^{-10}[5,6]$. Experimentally, the possible range for $f$ is bounded by $f \gtrsim 10^{6}-10^{9} \mathrm{GeV}$, via direct detection experiments [3] or stellar cooling bounds [7,8] (in the case of a coupling to the quark one even gets $f \gtrsim \mathcal{O}\left(10^{9}\right) \mathrm{GeV}$ [8]), although different bounds refer to coupling to different particles, namely photons, nucleons, quarks, or leptons. From Supernova SN 1987A [3,9-11], one gets $f \gtrsim \mathcal{O}\left(10^{8}\right) \mathrm{GeV}$, but such a bound should be considered less robust due to complicated SN physics. Moreover, the $\mathrm{SN}$ bound relies specifically on the axion-nucleons couplings, and so, it is somehow model dependent. In fact, the low energy effective Lagrangian for the QCD axion can arise from several UV completions, with different properties, leading to different effective couplings to the various standard model (SM) fields, including quarks and nucleons. The coherent oscillations of the axion can lead also to a

Published by the American Physical Society under the terms of the Creative Commons Attribution 4.0 International license. Further distribution of this work must maintain attribution to the author(s) and the published article's title, journal citation, and DOI. Funded by SCOAP. good candidate for Dark Matter, for $f \gtrsim 10^{11} \mathrm{GeV}$ [3,12-14].

Axions also naturally constitute a population of detectable hot relics from the early Universe [15-21] if the interactions that can produce them are fast enough at some stage of the radiation dominated Universe. A rather generic expectation is that axions can be produced in thermal abundance either via interactions with gluons $[18,19]$ or via direct interactions with the top quark [15,20], and they subsequently decouple and freeze out at a high temperature $T_{D}$ before the electroweak phase transition (EWPT).

Their relic abundance, in this case, would be proportional to the inverse of the effective number of degrees of freedom at the decoupling temperature $g_{*, D}$, and this would affect the energy density of relativistic particles at recombination, traditionally parametrized by an effective number of neutrinos $N_{\text {eff }}$, which can be tested by the CMB. Assuming only the existence of the SM plus the axion above the EWPT, the predicted change in $N_{\text {eff }}$ due to axions would be given by $\Delta N_{\text {eff }}=4 / 7\left(43 /\left(4 g_{*, D}\right)\right)^{4 / 3} \approx 0.027$ [22]. Remarkably, this is comparable to the forecasted sensitivity of future CMB-S4 experiments $[23,24]$. If there are additional degrees of freedom beyond the $\mathrm{SM}$ at such a high temperature $T_{D}$, this number would be smaller, so that this value is actually an upper bound. A possible thermalization at much later stages, below the QCD phase transition (QCDPT) via scattering with pions and nucleons, has been studied in $[16,17]$, concluding that this would require $f \lesssim \mathcal{O}\left(10^{6}\right) \mathrm{GeV}$, which is already in conflict with the astrophysical bounds [21]. 
In this Letter, we point out that if the axion is produced instead between the EWPT and the QCDPT, it can erase the previous prediction due to the high temperature thermalization before the EWPT, leading instead to larger values of $\Delta N_{\text {eff }}$, thus slightly easier to detect. Moreover, although there is room to have extra relativistic species at temperatures between the QCDPT and the EWPT, e.g., light relics or other weakly coupled particles, so far the standard model has passed precision tests with great success; so in this window of temperatures, the values of $\Delta N_{\text {eff }}$ can be seen more confidently as true predictions, as a function of $f$, instead of upper bounds. This happens due to direct couplings to various heavy quarks that are present in many axion models, as estimated already with dimensional analysis in [15]. Similar couplings were also studied in [23,25], although this was applied mostly to leptons, whose cross sections are suppressed due to the absence of coupling to gluons. Here, we explicitly compute the relevant cross sections, with $t-b$ and $c$ quarks, at temperatures sufficiently above the QCDPT, so that we can use a perturbative approach, which should be roughly correct as long as $\alpha_{s} \lesssim 1$. This calculation is timely because of the planned CMB-S4 experiments, which should be able to get close to the necessary sensitivity.

Axial couplings.-The axion has a Lagrangian that includes couplings with gauge bosons, and it can also include direct derivative couplings with fermions. We are particularly interested in the direct interaction with the different quarks $q_{i}$ of the SM,

$$
\mathcal{L}_{\text {int }}=\frac{\partial_{\mu} a}{2 f} \sum_{i} c_{i} \bar{q}_{i} \gamma^{\mu} \gamma^{5} q_{i}
$$

The coefficients $c_{i}$ are nonzero already at tree level, for example, in the Dine-Fischler-Srednicki-Zhitnitsky (DFSZ) models [26,27], and they are typically of order unity. (Here, we do not consider the case of couplings that mix different flavors. This has been considered in [23].) Note also that, in UV-completed axion models, the $c_{i}$ are proportional to the Peccei-Quinn charges over the domain wall number $N_{\mathrm{DW}}$ [22]. At high temperatures, above the EWPT, these types of couplings are the most efficient ones that can thermalize the axion [20], through the scatterings $t+h \rightarrow t+a$, $\bar{t}+h \rightarrow \bar{t}+a, t+\bar{t} \rightarrow h+a$, where $h$ is the Higgs particle. This is even larger than the scattering with gluons because it is not suppressed by $\alpha_{s} /(2 \pi)$, contrary to the axion-gluon coupling, and jointly with the fact that the top Yukawa coupling is $y_{t} \simeq 1$. By dimensional analysis, the rate of the axion-top-Higgs process goes as $\Gamma_{t-h} \propto y_{t}^{2} c_{t}^{2} T^{3} / f^{2}$, and so, it can be larger than the Hubble rate $H=\sqrt{g_{*} / 90} \pi T^{2} / M_{p}$, at sufficiently high temperatures, leading to an equilibrium distribution and subsequent decoupling when $\Gamma_{t-h} \simeq H$. Here, $M_{p}=2.4 \times 10^{18} \mathrm{GeV}$. Such a rate is larger than the one through gluons, which is given parametrically by $\Gamma_{g} \propto\left[\alpha_{s} /(2 \pi)\right]^{2} g_{s}^{2} T^{3} / f^{2}$, where $g_{s} \equiv \sqrt{4 \pi \alpha_{s}}$.

In this Letter, we will instead look at temperatures below the EWPT. At these energies, the Higgs particles are Boltzmann suppressed, so a much more relevant scattering is obtained by replacing the Higgs particle with a gluon, jointly with the fact that $g_{s} \gtrsim 1$. So, we will consider the processes

$q_{i}+g \rightarrow q_{i}+a, \quad \bar{q}_{i}+g \rightarrow \bar{q}_{i}+a, \quad q_{i}+\bar{q}_{i} \rightarrow g+a$.

A crucial feature of these cross sections is that, similarly to the case of $\Gamma_{t-h}$, they are proportional to the Yukawa coupling squared, $y_{i}^{2}$, which means to the squared mass of the quark, since the Higgs particle takes a vacuum expectation value (VEV) $v$ below the EWPT. This can be understood performing a field redefinition, as in [20], which erases the coupling of Eq. (1) and replaces it with a coupling of the type $y_{i} c_{i} / f a h \bar{q}_{i} \gamma^{5} q_{i}$. When the Higgs particle has a VEV, one can set $h=v$ so that the quark mass $\left(y_{i} v\right)^{2} / 2=m_{i}^{2}$ will actually appear in the cross sections. We work in the present Letter without performing such a redefinition, but we verify by explicit calculation that all cross sections indeed go as $m_{i}^{2}$.

For $T \gg m_{i}$, this represents a suppression. However, when $T \simeq m_{i}$, that is no longer the case, and such interactions dominate, e.g., over the ones with gluons.

For temperatures above the QCDPT, the cross sections $\sigma_{i}$ can be evaluated straightforwardly and they are rescaled for the various quarks $q_{i}$ simply by the respective $m_{i}^{2}$. In the end, we are interested in computing standard thermally averaged rates (see e.g., [18,22]),

$$
\Gamma_{i}=\frac{1}{n_{\mathrm{eq}}} \int \frac{d^{3} p_{a} d^{3} p_{b}}{(2 \pi)^{6} 4 E_{a} E_{b}} f_{a} f_{b}\left(\sigma_{i} 4 E_{a} E_{b}\right),
$$

where we integrate over the 3-momenta of the incoming states, $f_{a, b}$ are their thermal distributions (Bose-Einstein or Fermi-Dirac) and $n_{\text {eq }}$ is the axion equilibrium number density. Parametrically [15], one has $\Gamma_{i} \propto m_{i}^{2} c_{i}^{2} g_{s}^{2} T / f^{2}$ at $T \gtrsim m_{i}$. If, instead, $T \lesssim m_{i}$, a Boltzmann suppression arises due to $f_{a}$ and $f_{b}$, leading to

$$
\Gamma_{i} \propto e^{-\frac{m_{i}}{T}} \frac{m_{i}^{2} c_{i}^{2} g_{s}^{2} T}{f^{2}} .
$$

In order to find the precise numerical prefactor, we explicitly compute the cross sections. We simply use treelevel diagrams, also adding thermal masses in the kinematics; for a more refined treatment, one could evaluate in thermal field theory the axion self-energy, as in $[19,20]$, although we expect this will lead only to minor corrections. Comparing the scattering rates $\Gamma_{i}$ with $H$ allows us to know, at a given temperature, at which value of $f$ the axion 
is in thermal equilibrium. More precisely, we check whether $\Gamma_{i} /(3 H)>1$. At a given $T$, the most important channel is the scattering with the heaviest quark, which is not yet Boltzmann suppressed. Thus, the rates $\Gamma_{i}$ are maximal around $T \simeq m_{i}$.

For the first two processes in Eq. (2), the cross section can be quite simply expressed under the approximation of massless gluons (i.e., neglecting thermal masses) as

$4 \sigma_{i}^{(1)} v E_{a} E_{b}=\frac{4 c_{i}^{2} g_{s}^{2} m_{i}^{2}}{\pi f^{2}}\left[\tanh ^{-1}\left(\frac{p}{E}\right)-\frac{p(2 p+E)}{(p+E)^{2}}\right]$,

where $p$ and $E$ are the momentum and energy of the quark, respectively, in the center of mass frame. Instead, the cross section for pair annihilation takes a simple form, even including the gluon thermal mass $m_{g, t h}$,

$$
4 \sigma_{i}^{(2)} v E_{a} E_{b}=\frac{2 c_{i}^{2} g_{s}^{2} m_{i}^{2}}{\pi f^{2}} \frac{4 E^{2}-m_{g, \mathrm{th}}^{2}}{E p} \tanh ^{-1}\left(\frac{p}{E}\right) .
$$

In our final results, we will include gluon thermal masses [28],

$$
m_{g, \mathrm{th}}^{2}=\left(\frac{N_{c}}{6}+\frac{N_{f}}{12}\right) g_{s}^{2} T^{2}
$$

where $N_{c}$ and $N_{f}$ are respectively the number of colors and flavors. We will also use in the quark dispersion relations an overall effective mass, which includes both the standard mass $m_{i} \equiv y_{i} v / \sqrt{2}$ and the thermal mass [28]

$$
m_{i, \mathrm{eff}}^{2}=m_{i, \mathrm{th}}^{2}+m_{i}^{2}=\frac{N_{f}}{8} g_{s}^{2} T^{2}+m_{i}^{2} .
$$

Note however that, while the thermal masses modify the kinematics, they cannot enter in the overall prefactor $y_{i}^{2} v^{2}$ that appears in the cross sections, since the thermal masses change the dispersion relations but preserve chirality [28]. (One could check this by an explicit calculation using the thermal one-loop fermionic propagator, as was done, e.g., in a different context in [29], Appendix C.1. However, here for simplicity, we implement this by including the thermal masses in the kinematics, but not in the prefactor.) This can also be seen by performing the field redefinition of [20] again, which shows that the cross section must vanish for $y_{i} \rightarrow 0$, and therefore, there cannot be a contribution to the prefactor proportional to $m_{i, \mathrm{th}}$, which is independent of $y_{i}$. The inclusion of gluon thermal masses does not dramatically change the result, but it is important conceptually because it kinematically forbids processes with emission of many gluons, which could be otherwise dominant since we have $g_{s}>1$. Finally, we evaluate the couplings inside the integral at the center-of-mass energy $g_{s}(2 E)$ by using a simple one-loop renormalization group equation running.
We find that the rates are well approximated by the following fitting functions $\Gamma_{i}=e^{-\left(m_{i} / T\right)} m_{i}^{2} c_{i}^{2} g_{s}^{2} T /$ $f^{2}\left[A_{i}+B_{i} \log \left(T / M_{Z}\right)\right]$, where $M_{Z}$ is the $Z$-boson mass and $\left(A_{t}, B_{t}\right)=\left(1 \times 10^{-2},-10^{-4}\right),\left(A_{b}, B_{b}\right)=\left(1.1 \times 10^{-2},-10^{-4}\right)$ and $\left(A_{c}, B_{c}\right)=\left(1.3 \times 10^{-2},-2 \times 10^{-4}\right)$.

Scattering rates and $N_{\text {eff }}$ : Results.-In Fig. 1, we plot $\Gamma_{i} /(3 H)$ as a function of $T$, for the scatterings involving $t$, $b$, and $c$ quarks (we do not consider the $s$ quark, since the calculation would be unreliable, being too close to the QCDPT), plotting each curve at two different values of $f / c_{i}$. Note that, what determines the final axion abundance is the decoupling temperature. In all our plots, we use the one-loop analytical expression for the temperature dependence of the Higgs VEV $v(T)$, which is important close to $100 \mathrm{GeV}$, especially in the case of the $t$ quark. At large values of $f$, say $f / c_{t} \gtrsim 10^{9} \mathrm{GeV}$, the axion can become thermal via the top-Higgs scatterings (we use as a crude estimate the expression given in [20], times a Boltzmann factor $e^{-m_{t} / T}$, although such results were derived for massless particles, with no Higgs VEV), already at $T \gtrsim \mathcal{O}(100) \mathrm{GeV}$. However, the axion can also be produced below the EWPT through the scatterings with the $t$ quark, Eq. (2) for $f / c_{t} \lesssim 10^{9} \mathrm{GeV}$ or, for an even smaller $f$, through scatterings with the $b$ or $c$ quarks.

The important consequence of axion production is the observable effect on the effective number of relativistic degrees of freedom $N_{\text {eff }}$. In the SM, this is predicted to be $[30,31] N_{\text {eff }}=3.045$, which tells us that the only relativistic species at recombination, apart from photons, are the three neutrinos. Any deviation from this would tell us something new about the Universe, in particular, about light relics and/or additional degrees of freedom.

A simple way to estimate $\Delta N_{\text {eff }}$ would be to simply read the decoupling temperature from Fig. 1 and to evaluate $g_{* D}$, i.e., from [32]. In order to compute $\Delta N_{\text {eff }}$ more precisely, we solve the Boltzmann equation (this equation is valid in the Boltzmann approximation) for the axion number density $n_{a}$,

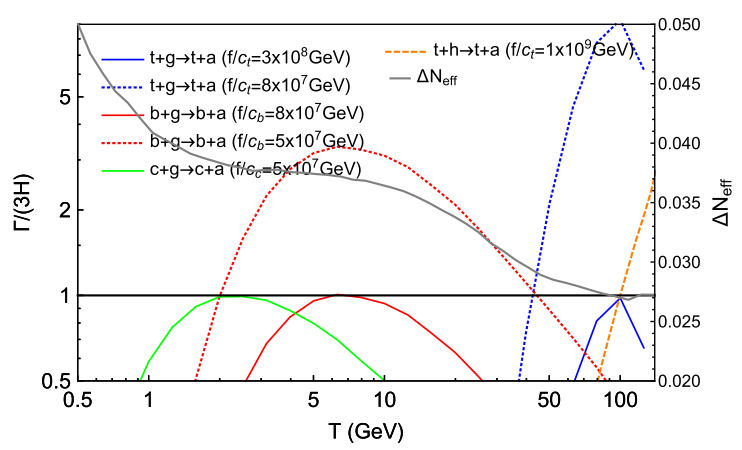

FIG. 1. $\Gamma_{i} /(3 H)$ vs the temperature associated with the axion production via scatterings with different quarks and gluons. Each process is evaluated at values of $f / c_{i}$, chosen to be the minimal ones, such that $\Gamma_{i} /(3 H)=1$. The orange line uses the result derived in [20]. 


$$
\frac{d n_{a}}{d t}+3 H n_{a}=\Gamma_{i}\left(n_{a}-n_{\mathrm{eq}}\right) .
$$

This equation can also be rewritten in terms of the abundance $Y \equiv n_{a} / s$,

$$
s H z \frac{d Y}{d z}=\gamma_{i}\left(1-\frac{Y}{Y_{\mathrm{eq}}}\right),
$$

where $z \equiv m_{i} / T, \quad Y_{\text {eq }} \equiv n_{\text {eq }} / s, \quad \gamma_{i} \equiv \Gamma_{i} n_{\text {eq }}, \quad$ and $\quad s \equiv$ $2 \pi^{2} g_{*} T^{3} / 45$ is the entropy density. Assuming a constant $g_{*}$, there is an analytical solution. Parametrizing $\gamma_{i} \equiv \gamma_{m_{i}} z^{-4} e^{-z}, H \equiv H_{m_{i}} z^{-2}$ and $s \equiv s_{m_{i}} z^{-3}$, and imposing $Y=0$ at $z \rightarrow 0$, we get:

$$
Y=Y^{\mathrm{eq}}\left[1-e^{-\left(1-e^{-z}\right) r}\right],
$$

where $r \equiv \gamma_{m_{i}} /\left(H_{m_{i}} s_{m_{i}} Y^{\mathrm{eq}}\right)=(\Gamma / H)_{T=m_{i}}$. So, the final abundance is $Y(z=\infty)=Y^{\mathrm{eq}}\left(1-e^{-r}\right)$, which corresponds to a change in $N_{\text {eff }}$ given by

$$
\Delta N_{\mathrm{eff}}=\frac{4}{7}\left(\frac{43}{4 g_{*, D}}\right)^{\frac{4}{3}}\left[1-e^{-\left.(\Gamma / H)\right|_{T=m_{i}}}\right]
$$

However, $g_{*}$ has a nontrivial dependence with $T$ [32], so we need to solve the Boltzmann equation numerically. (Other temperature dependences that we also included numerically are: the running of $g_{s}$, the Higgs $\operatorname{VEV} v(T)$, and the logarithmic part of the rates $\Gamma_{i}$.) We stop the simulation at $T=0.5 \mathrm{GeV}$, when $\alpha_{s}=1$. In the case of $t$, the results are insensitive to this cutoff. In the case of $b$, the results are also insensitive to it, provided $f / c_{b} \gtrsim 10^{7} \mathrm{GeV}$. However, if the axion production is still nonzero at $T \lesssim 0.5 \mathrm{GeV}$, as happens for the $b$ when $f / c_{b} \lesssim 10^{7} \mathrm{GeV}$, and for the $c$ when $f / c_{c} \lesssim 2 \times 10^{8} \mathrm{GeV}$, that can affect $\Delta N_{\text {eff }}$, given that $g_{*}(T)$ varies rapidly in that region. To get a good estimate, one would need more refined techniques, which we leave for future work.

We plot the results in Fig. 2, showing the predicted $\Delta N_{\text {eff }}$ as a function of $f / c_{i}$ for $i=t, b, c$. As anticipated from Fig. 1, thermalization via top scatterings happens when $f / c_{t} \approx 4 \times 10^{8} \mathrm{GeV}$, leading to $\Delta N_{\text {eff }} \approx 0.03$. For a smaller $f / c_{t}$, the axion remains thermal for a longer time, leading to a larger $\Delta N_{\text {eff }}$. For example, at $10^{6} \mathrm{GeV} \leq f / c_{t} \leq 10^{7} \mathrm{GeV}$, $\Delta N_{\text {eff }}=0.035-0.036$. Note, however, that such values of the axial couplings to the top might already be excluded by stellar cooling constraints, $f / c_{t} \gtrsim 2.4 \times 10^{9} \mathrm{GeV}$ [8].

Similarly, the scatterings with the bottom are able to thermalize the axion at $f / c_{b} \approx(2-3) \times 10^{8} \mathrm{GeV}$, leading to $\Delta N_{\text {eff }}=0.038$. In this case, at $f / c_{b}=10^{7} \mathrm{GeV}$, one has $\Delta N_{\text {eff }}=0.047$, which is interestingly close to the forecasted $2 \sigma$ contour of CMB-S4 [24]. For $f / c_{b}$ below $10^{7} \mathrm{GeV}$, we encounter the problem that we have stressed before: the axion decouples at $T \lesssim 0.5 \mathrm{GeV}$, where the methods used here break down. To see this sensitivity, we

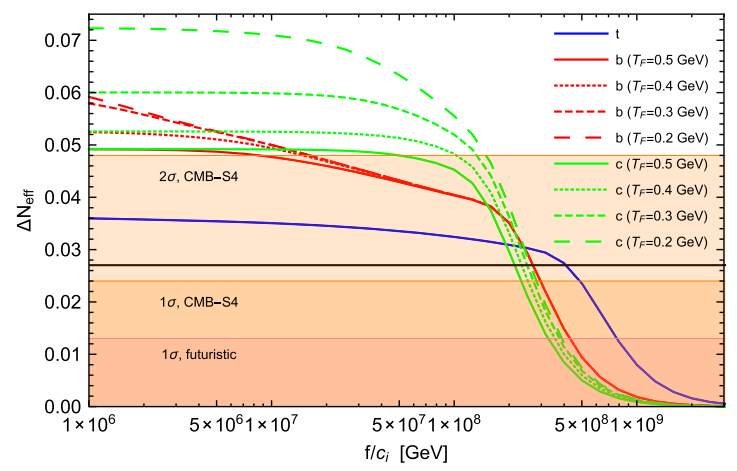

FIG. 2. $\Delta N_{\text {eff }}$ as a function of $f / c_{i}$. The green, red, and blue labels correspond to the predictions for the charm, bottom, and top particle, respectively. The orange bands represent the $1 \sigma$ and $2 \sigma$ forecasted contours of CMBS 4 , plus a more futuristic $1 \sigma$ band, according to [24]. See also [33] for similar forecasts combining CMB-S4 and large scale structure.

have plotted 4 red lines. The lowest one corresponds to a case where we stop the simulation at a final temperature $T_{F}=0.5 \mathrm{GeV}$. Such a line should be seen as a lower bound on $\Delta N_{\text {eff }}$ at any given value of $f / c_{b}$. The other three lines correspond to cases where we fix $\alpha_{s}=1$ for $T<$ $0.5 \mathrm{GeV}$ by hand and then run the simulation down to lower temperatures $T_{F}=0.4,0.3,0.2 \mathrm{GeV}$, respectively. The lowest red line gives a lower bound on $\Delta N_{\text {eff }}$, which at $10^{6} \mathrm{GeV} \leq f / c_{b} \leq 10^{7} \mathrm{GeV}$, is $\Delta N_{\text {eff }}>0.049$.

Regarding the scatterings, with the charm they are able to thermalize around $f / c_{c} \lesssim 10^{8} \mathrm{GeV}$. This case is affected, to a greater extent, by the same uncertainty due to strong coupling, and the final predictions turn out to be very sensitive to what happens below $0.5 \mathrm{GeV}$. Also, in this case, we plot 4 lines to show the sensitivity to $T_{F}$, and again, from the lowest green line we can give a lower bound on $\Delta N_{\text {eff }}$, which at $f / c_{c}<10^{8} \mathrm{GeV}$ is $\Delta N_{\text {eff }}>0.045$. Such values, obtained close to the strong coupling region, are of great interest, since they could be potentially measurable at $2 \sigma$ by CMB-S4. However, a dedicated study of such cases would be needed, using more adequate methods to treat the axion decoupling around the QCDPT.

Finally, note that in all cases, even if the axion does not thermalize completely, it can still have a non-negligible abundance, and even give $\Delta N_{\text {eff }}>0.027$.

In conclusion, we have shown that, in addition to the possible thermalization at $f \gtrsim 10^{9} \mathrm{GeV}$, before the EWPT via gluons $[18,19]$ or axion-top-Higgs scatterings [20], the axion can be produced at intermediate energy scales between the EWPT and the QCDPT via direct couplings to heavy quarks. Such couplings are present in various models, such as DFSZ, at tree level, and thus, they are enhanced compared to interactions with gluons, which are generated at one loop. Clearly, because we are probing lower energies, this happens in a lower range of $f$, which, by solving the associated Boltzmann equation, turns out to be $f / c_{i} \lesssim 10^{9} \mathrm{GeV}$. This window can be viable if SN 
bounds are not so robust [11] or, as pointed out in [34], in DFSZ-like axion models that can avoid SN bounds, because of suppressed coupling to light quarks and thus to nucleons. Interestingly in the same window one could also explain the hints of extra stellar energy losses, as suggested in [11]. (Note also that, although this region does not overlap with the standard window of axion dark matter, it may be possible to obtain QCD axion DM even at such small $f$, via domain wall decay $[11,35,36]$ or via resonant production of axions at the PQ phase transition [37], although in such cases one should check whether the scatterings we consider can destroy the condensate. Furthermore our window of $f$ can also be relevant in the context of axion-like particles, as in [38]).

Axion production and decoupling happen at different thresholds $T_{i}$, close to the heavy quark masses $m_{i}$, leading to different predictions on $\Delta N_{\text {eff }}$ as a function of $f / c_{i}$, shown in Fig. 2, which contains our main results. We stress that such predictions are not upper bounds, but can be precisely given, as far as we know, the number of degrees of freedom $g_{*, D}$ and the dynamics at such energies. For the top and bottom our main predictions $\left(0.027 \leq \Delta N_{\text {eff }} \leq 0.036\right.$ for $10^{6} \mathrm{GeV} \lesssim f / c_{t} \lesssim 4 \times 10^{8} \mathrm{GeV}$ and $0.027 \leq \Delta N_{\text {eff }} \leq$ 0.047 for $10^{7} \mathrm{GeV} \lesssim f / c_{b} \lesssim 3 \times 10^{8} \mathrm{GeV}$ ) are quite under control, while for the $c$ quark, since axion production is too close to the QCD phase transition, we only have a lower bound $\Delta N_{\text {eff }} \geq 0.045$ (at $f / c_{c} \lesssim 10^{8} \mathrm{GeV}$ ), which nonetheless might represent the largest signal for a detection.

These observations open a new window to test the QCD axion. At the same time, if the axion exists in this range of $f$, this would allow us to probe the early Universe at energies so far unexplored in cosmology, between the QCD and the EW phase transitions. If the axion could be detected directly within such a window for $f$, e.g., with the CAST [39] or IAXO Collaboration [40-42] experiments, our conclusions would make it possible to confirm if our expectations about the total particle content and the thermal history at such temperatures are indeed correct.

We thank Luca di Luzio for initial discussions about the project, Federico Mescia, Alberto Salvio, Francesco d'Eramo, Fernando Arias-Aragon, Andreas Ringwald, and Javier Redondo for enlightening discussions and useful comments. This work is supported by the Grants No. EC FPA2010-20807-C02-02, No. AGAUR 2009-SGR-168, ERC Starting Grant No. HoloLHC-306605, and by the Spanish MINECO under MDM-2014-0369 of ICCUB (Unidad de Excelencia "Maria de Maeztu").

*rferreira@icc.ub.edu †notari@fqa.ub.edu

[1] R. D. Peccei and H. R. Quinn, Constraints imposed by $C P$ conservation in the presence of instantons, Phys. Rev. D 16, 1791 (1977).
[2] R. D. Peccei and H. R. Quinn, $C P$ Conservation in the Presence of Instantons, Phys. Rev. Lett. 38, 1440 (1977).

[3] C. Patrignani et al. (Particle Data Group Collaboration), Review of particle physics, Chin. Phys. C 40, 100001 (2016).

[4] H.-Y. Cheng, The strong $C P$ problem revisited, Phys. Rep. 158, 1 (1988).

[5] C. A. Baker, D. D. Doyle, P. Geltenbort, K. Green, M. G. D. vanderGrinten, P. G. Harris, P. Iaydjiev, S. N. Ivanov, D. J. R. May, J. M. Pendlebury, J. D. Richardson, D. Shiers, and K. F. Smith, An Improved Experimental Limit on the Electric Dipole Moment of the Neutron, Phys. Rev. Lett. 97, 131801 (2006).

[6] J. M. Pendlebury et al., Revised experimental upper limit on the electric dipole moment of the neutron, Phys. Rev. D 92, 092003 (2015).

[7] B. M. S. Hansen, H. Richer, J. Kalirai, R. Goldsbury, S. Frewen, and J. Heyl, Constraining neutrino cooling using the hot white dwarf luminosity function in the globular cluster 47 tucanae, Astrophys. J. 809, 141 (2015).

[8] J. L. Feng, T. Moroi, H. Murayama, and E. Schnapka, Third generation familons, b factories, and neutrino cosmology, Phys. Rev. D 57, 5875 (1998).

[9] G. G. Raffelt, Astrophysical axion bounds, Lect. Notes Phys. 741, 51 (2008).

[10] T. Fischer, S. Chakraborty, M. Giannotti, A. Mirizzi, A. Payez, and A. Ringwald, Probing axions with the neutrino signal from the next galactic supernova, Phys. Rev. D 94, 085012 (2016).

[11] M. Giannotti, I. G. Irastorza, J. Redondo, A. Ringwald, and K. Saikawa, Stellar recipes for axion hunters, J. Cosmol. Astropart. Phys. 10 (2017) 010.

[12] J. Preskill, M. B. Wise, and F. Wilczek, Cosmology of the Invisible Axion, Phys. Lett. 120B, 127 (1983).

[13] L. F. Abbott and P. Sikivie, A cosmological bound on the invisible axion, Phys. Lett. 120B, 133 (1983).

[14] M. Dine and W. Fischler, The not so harmless axion, Phys. Lett. 120B, 137 (1983).

[15] M. S. Turner, Thermal Production of Not SO Invisible Axions in the Early Universe, Phys. Rev. Lett. 59, 2489 (1987); Erratum, 60, 1101(E) (1988).

[16] Z. G. Berezhiani, A. S. Sakharov, and M. Yu. Khlopov, Primordial background of cosmological axions, Yad. Fiz. 55, 1918 (1992) [Sov. J. Nucl. Phys. 55, 1063 (1992)].

[17] S. Chang and K. Choi, Hadronic axion window and the big bang nucleosynthesis, Phys. Lett. B 316, 51 (1993).

[18] E. Masso, F. Rota, and G. Zsembinszki, On axion thermalization in the early universe, Phys. Rev. D 66, 023004 (2002)

[19] P. Graf and F. D. Steffen, Thermal axion production in the primordial quark-gluon plasma, Phys. Rev. D 83, 075011 (2011).

[20] A. Salvio, A. Strumia, and W. Xue, Thermal axion production, J. Cosmol. Astropart. Phys. 01 (2014) 011.

[21] C. Brust, D. E. Kaplan, and M. T. Walters, New light species and the CMB, J. High Energy Phys. 12 (2013) 058.

[22] E. W. Kolb and M. S. Turner, The Early Universe, Frontiers in physics Vol. 69 (Perseus, 1994).

[23] D. Baumann, D. Green, and B. Wallisch, New Target for Cosmic Axion Searches, Phys. Rev. Lett. 117, 171301 (2016). 
[24] K. N. Abazajian et al. (CMB-S4 Collaboration), CMB-S4 Science Book, First Edition, arXiv:1610.02743.

[25] D. Cadamuro, S. Hannestad, G. Raffelt, and J. Redondo, Cosmological bounds on sub-MeV mass axions, J. Cosmol. Astropart. Phys. 02 (2011) 003.

[26] A. R. Zhitnitsky, On possible suppression of the axion hadron interactions. (In Russian), Yad. Fiz. 31, 497 (1980) [Sov. J. Nucl. Phys. 31, 260 (1980)].

[27] M. Dine, W. Fischler, and M. Srednicki, A simple solution to the strong $C P$ problem with a harmless axion, Phys. Lett. 104B, 199 (1981).

[28] M. L. Bellac, Thermal Field Theory (Cambridge University Press, Cambridge, 2011).

[29] G. F. Giudice, A. Notari, M. Raidal, A. Riotto, and A. Strumia, Towards a complete theory of thermal leptogenesis in the SM and MSSM, Nucl. Phys. B685, 89 (2004).

[30] G. Mangano, G. Miele, S. Pastor, T. Pinto, O. Pisanti, and P. D. Serpico, Relic neutrino decoupling including flavor oscillations, Nucl. Phys. B729, 221 (2005).

[31] P. F. de Salas and S. Pastor, Relic neutrino decoupling with flavour oscillations revisited, J. Cosmol. Astropart. Phys. 07 (2016) 051.

[32] S. Borsanyi et al., Calculation of the axion mass based on high-temperature lattice quantum chromodynamics, Nature (London) 539, 69 (2016).
[33] D. Baumann, D. Green, and B. Wallisch, Searching for Light Relics with Large-Scale Structure, arXiv:1712.08067.

[34] L. Di Luzio, F. Mescia, E. Nardi, P. Panci, and R. Ziegler, The Astrophobic Axion, arXiv:1712.04940.

[35] M. Kawasaki, K. Saikawa, and T. Sekiguchi, Axion dark matter from topological defects, Phys. Rev. D 91, 065014 (2015).

[36] A. Ringwald and K. Saikawa, Axion dark matter in the postinflationary Peccei-Quinn symmetry breaking scenario, Phys. Rev. D 93, 085031 (2016); Publisher's Note, 94, 049908(A) (2016).

[37] R. T. Co, L. J. Hall, and K. Harigaya, QCD Axion Dark Matter with a Small Decay Constant, arXiv:1711.10486.

[38] R. Daido, F. Takahashi, and W. Yin, The ALP miracle: Unified inflaton and dark matter, J. Cosmol. Astropart. Phys. 05 (2017) 044.

[39] E. Arik et al. (CAST Collaboration), Probing eV-scale axions with CAST, J. Cosmol. Astropart. Phys. 02 (2009) 008.

[40] E. Armengaud et al., Conceptual design of the international axion observatory (IAXO), J. Instrum. 9, T05002 (2014).

[41] I. Irastorza et al. (IAXO Collaboration), The International Axion Observatory IAXO, Letter of Intent to the CERN SPS committee.

[42] I. G. Irastorza et al., Towards a new generation axion helioscope, J. Cosmol. Astropart. Phys. 06 (2011) 013. 